fragmentum 20

História das ideias linguisticas

Apresentação de

Cláadia Castellanos Pfeiffer (Unicamp/Labearb)

Larissa Montagner Cervo (LabCorpus)

(Org.) 
Fragmentum / Universidade Federal de Santa Maria.

Centro de Artes e Letras. Programa de Pós-Graduação em Letras. Laboratório Corpus. - N.1 (set. 2001) -

Santa Maria, 2001 -

Trimestral

ISSN 1519-9894

N. 20 (jan.-mar. 2009)

CDU 801

Ficha catalográfica elaborada por Alenir Inácio Goularte CRB-10/990 Biblioteca Central da UFSM

EDITORA PPGL

Impresso na IMPRENSA UNIVERSITÁRIA

UFSM - Santa Maria - RS

Maio de 2010 


\title{
História das ideias linguisticas
}

\author{
Apresentação de \\ Cláudia Castellanos Pfeiffer (Unicamp/Labeurb) \\ Larissa Montagner Cervo (LabCorpus) \\ (Org.)
}

FRAGMENTUM No 20, JAN./MAR. DE 2009 Laboratório Corpus Programa de Pós-Graduação em Letras

Centro de Artes e Letras Universidade Federal de Santa Maria Santa Maria, RS

ISSN 1519-989 
fragmentum

Revista do Laboratório Corpus do Programa de Pós-Graduação em Letras UFSM

Reitor

Felipe Martins Müller

Diretor do Centro de Artes e Letras

Edemur Casanova

Coordenadora do Programa de Pós-Graduação em Letras

Amanda Eloina Scherer

Coordenadora Geral do LabCorpus

Amanda Eloina Scherer

Coordenador Executivo do LabCorpus

Pedro Brum Santos

Coordenadora Acadêmico-Cientifica do LabCorpus

VerliPetri

Assistente de Pesquisa do LabCorpus

Rejane Arce Vargas

Pesquisadores do LabCorpus

Amanda Eloína Scherer

Eliana Rosa Sturza

Graziela de Lucci de Angelo

Marcia Cristina Corrêa

Mirian Rose Brum-de-Paula

Pedro Brum Santos

Silvia Paraense 
Tania Regina Taschetto

Vera Lúcía Lenz Vianna

VerliPetri

\section{Corpo Editorial}

Amanda Eloina Scherer (Presidente)

Pedro Brum Santos

VerliPetri

\section{Comitê Editorial}

Ana Zandwais - UFRGS

Beatriz Eckert-Hoff - UNITACVUniAnchieta

Bethanía Mariani - UFF

Carme Schons - UPF

Christian Leray - Rennes 2 - França

Colette Noyau - París X - França

Cristiane Dias - UNICAMP

Eduardo Guimarães - UNICAMP

EniPuccinelli Orlandi- UNICAMP

Flávio Loureiro Chaves - UFRGS

Gema Sanz Espinar - Universidad de Madrid - Espanha

Gesualda Rasía - LIFPR

Gladys B. Morales - Universidad Nacional de Río Cuarto - Argentína

Hélène Leclercq - IUFM - Université de Franche-Comté

Héliane Kohler - Université de Franche-Comté

José Horta Nunes - LINESP

Luiz Francisco Dias - UFMG

Maria Cleci Venturini - UNICENTRO

María Elena Villecco - Universidad de Tucumán - Argentina

María José R. Faria Coracini - UNICAMP

María Josefina Garat - Universidad de Tucumán - Argentina

Véronique Daleth - USP 


\section{Capa e Projeto Gráfico Originais}

Mirian Rose Brum-de-Paula

Projeto Gráfico

Rejane Arce Vargas

\section{Diagramação}

Juciele Pereira Días

Revisão de linguagem

Larissa Montagner Cervo

Supervisão da publicação

Amanda Eloina Scherer

Periodicidade

Trimestral

\section{Editora}

Programa de Pós-Graduação em Letras

Universidade Federal de Santa Maria

Prédio 16, CE, sala $3222-B l o c o A 2$

Campus Universitário Camobi

$97105-900$ - Santa Maria, RS - Brasil

Fones: 0xx 5532208359 ; Oxx 5532208025

Email: ppgl@mail.ufsm.br

Site : www.ufsm.br/ppgletras

\section{Data da Efetiva Círculação}

Fevereiro de 2010

Solicita-se permuta 